\title{
ORIENTAÇÕES MOTIVACIONAIS, CRENÇAS EDUCACIONAIS E DESEMPENHO ESCOLAR DE ESTUDANTES DO ENSINO FUNDAMENTAL ${ }^{1}$
}

\author{
Mirella Lopez Martini Fernandes Paiva* \\ Evely Boruchovitch"
}

\begin{abstract}
RESUMO. Este trabalho teve como objetivo investigar as orientações motivacionais, as crenças sobre esforço, inteligência e sorte e sobre as condições necessárias para a obtenção do sucesso na escola e as expectativas futuras quanto à superação de um desempenho acadêmico ruim, de 120 alunos do Ensino Fundamental. Examinou, ainda, as relações dessas variáveis entre si e com o desempenho acadêmico, o gênero e a série escolar. Os dados foram obtidos por meio da aplicação individual da Entrevista Estruturada de Crenças Educacionais e Orientações Motivacionais do Aluno e do Teste de Desempenho Escolar de Stein. Os resultados indicam um predomínio da motivação intrínseca e um padrão de crenças altamente apropriado à motivação e à aprendizagem. As crenças de meninos, de alunos repetentes e daqueles extrinsecamente motivados merecem atenção especial, dadas as implicações educacionais envolvidas e a necessidade de planejamento de intervenções mais focalizadas.
\end{abstract}

Palavras-chave: Orientações motivacionais; crenças educacionais; desempenho acadêmico.

\section{MOTIVATIONAL ORIENTATION, EDUCATIONAL BELIEFS AND ACADEMIC ACHIEVEMENT OF ELEMENTARY SCHOOL STUDENTS}

\begin{abstract}
The main goal of the present study was to investigate the motivational orientations and beliefs about effort, intelligence, luck, and about the necessary conditions to achieve success in school as well as the expectations to overcome a poor academic performance in a sample of 120 students from elementary school. The relationship between such variables, and their correlation to academic performance, gender and school grades was also analyzed. Data were acquired by applying the Structured Interview of Educational Beliefs and Motivational Orientation for Student and School Achievement Test from Stein in an individual basis. Results indicate a predominance of intrinsic motivation and a highly appropriate beliefs pattern to motivation and learning. Given the educational implications involved, boys, repeater and extrinsically motivated student beliefs must be carefully taken into account to better plan more focused interventions.
\end{abstract}

Key words: Motivational orientation; educational beliefs; academic achievement.

\section{ORIENTACIÓN MOTIVACIONAL, CREENCIAS EDUCATIVAS Y LOS LOGROS ACADÉMICOS DE LOS ESTUDIANTES DE LA ESCUELA PRIMARIA}

\begin{abstract}
RESUMEN. Este trabajo tuvo como objetivo investigar las orientaciones motivacionales, las creencias sobre esfuerzo, inteligencia y suerte, sobre las condiciones necesarias para la obtención del éxito en la escuela y las expectativas futuras en cuanto a la superación de un desempeño académico malo, de 120 alumnos de la Enseñanza Fundamental. Examinó, aún, las relaciones de esas variables entre sí y con el desempeño académico, género y serie escolar. Los datos fueron obtenidos por medio de la aplicación individual de la Entrevista Estructurada de Creencias Educacionales y Orientaciones Motivacionales del Alumno y del Test de Desempeño Escolar de Stein. Los resultados indicaron un predominio de la motivación intrínseca y un patrón de creencias altamente apropiado a la motivación y aprendizaje. Las creencias de chicos, alumnos repitentes y extrínsecamente motivados merecen atención especial, dadas las implicaciones educacionales envueltas y la necesidad de planes de intervención más focalizados.
\end{abstract}

Palabras-clave: Orientaciones motivacionales; creencias educacionales; desempeño académico.

Apoio: FAPESP, CNPq e Capes.

Psicóloga. Mestre em Educação e Doutora em Ciências. Membro do grupo de pesquisa Relações Interpessoais e Habilidades Sociais (RIHS) e do Núcleo de Estudo de Motivação Acadêmica (NEMA) da Universidade Federal de São Carlos/UFSCar.

\# Psicóloga. PhD em Educação. Professora Livre-Docente do Departamento de Psicologia Educacional da Faculdade de Educação da Universidade de Campinas, membro do Grupo de Estudos e Pesquisas em Psicopedagogia (GEPESP) - UNICAMP 
Nos últimos anos a pesquisa psicológica e educacional tem priorizado o estudo sobre a motivação dos alunos, dada a sua relevância para o processo de ensino-aprendizagem e para o desenvolvimento socioemocional e cognitivo dos discentes (Bzuneck, 2004). Os avanços nessa área advêm principalmente do conhecimento obtido pelos estudos que se baseiam nas teorias sociocognitivistas, entre as quais uma abordagem que se tem mostrado bastante útil é a Teoria da Autodeterminação -SDT (Ryan \& Deci, 2000; Reeve, Ryan \& Deci, 2004).

De acordo com Reeve, Ryan e Deci (2004), a SDT é uma macroteoria da motivação, pois oferece uma abordagem de compreensão e maximização da motivação dos alunos. A SDT assume que todos os alunos apresentam uma orientação geral para o crescimento e também necessidades psicológicas inatas que, juntas, constituem a base para a motivação autônoma e para o desenvolvimento psicológico saudável. Como uma macroteoria, a SDT foi elaborada a partir de quatro miniteorias complementares e inter-relacionadas: a Teoria das Necessidades Básicas, a Teoria da Avaliação Cognitiva, a Teoria da Integração Organísmica e a Teoria das Orientações Causais.

A Teoria da Integração Organísmica considera que as regulações externas podem ser internalizadas e, a partir dessa internalização, o indivíduo pode desenvolver as formas autorreguladas de motivação extrínseca. Propõe a existência de um continuum, que vai desde a desmotivação e as formas autorreguladas de motivação extrínseca até a motivação intrínseca, sendo um referencial teórico muito valioso para os estudos relacionados ao contexto acadêmico (Boruchovitch, 2008).

Em linhas gerais, pode-se dizer que alunos intrinsecamente motivados se envolvem e permanecem na tarefa pelo próprio prazer, desafio, curiosidade e interesse que a atividade lhes desperta, enquanto alunos extrinsecamente motivados cumprem as tarefas para obterem recompensas externas e/ou demonstrar suas competências e capacidades às outras pessoas (Ryan \& Deci, 2000).

Estudos revelam que os alunos intrinsecamente motivados apresentam melhores índices de desempenho acadêmico, são menos ansiosos, são avaliados pelos professores como sendo os alunos mais esforçados e que melhor aprendem, além de demonstrarem índices elevados de autoconceito acadêmico e autoconceito geral (Gottfried, 1990; 1985; Gottfried \& Gottfried, 2004; Gottfried, Gottfried \& Fleming, 2001; Gottfried, Gottfried, Cook \& Morris, 2005; Gottfried, Marcoulides, Gottfried, Oliver \& Guerin, 2007).

Muito embora seja consenso entre os pesquisadores que a motivação intrínseca é mais apropriada para a aprendizagem, se comparada à motivação extrínseca, alguns estudiosos apontam a necessidade de essas duas orientações motivacionais coexistirem e serem complementares (Guimarães, 2004; Ryan \& Deci, 2000). É necessário reconhecer que nem todas as situações educacionais garantem que os alunos possam engajar-se em tarefas acadêmicas unicamente por fatores intrínsecos, por isso os fatores internos e externos devem inter-relacionar-se para produzir um desempenho orientado para a motivação intrínseca do aluno (Lepper, Corpus \& Iyengar 2005).

As teorias sociocognitivas da motivação afirmam, ainda, que a motivação e o desempenho acadêmico dos alunos baseiam-se nos processos de mediação cognitiva, constituídos pelas crenças e pelos valores conscientes do aluno (Bandura, 1982). Essas crenças funcionam como um filtro pelo qual o aluno interpreta a si mesmo e a realidade escolar, e influenciam fortemente suas ações, suas tomadas de decisão e seu planejamento futuro.

Entre o conjunto de crenças relacionadas à aprendizagem e ao desempenho dos alunos destaca-se a compreensão desses alunos acerca do papel da inteligência/capacidade, do esforço e da sorte no processo de ensino-aprendizagem (Weiner, 1985). Segundo Weiner (1985), inteligência/capacidade, esforço, sorte e dificuldades da tarefa são as atribuições mais frequentemente utilizadas pelos indivíduos como explicação para os resultados de sucesso ou fracasso em tarefas acadêmicas. Dependendo da forma como são compreendidas, elas podem influenciar de diferentes maneiras o comportamento subsequente do indivíduo.

De acordo com Dweck e Bempechat (1983), as pessoas desenvolvem teorias pessoais acerca da sua própria inteligência, as quais trazem implícitos dois tipos de crença: a inteligência tida como um traço imutável e fixo ou a visão incremental de que a inteligência pode ser desenvolvida e ampliada mediante o esforço e a prática. Blumenfield, Pintrich e Hamilton (1986) encontraram diferenças de desenvolvimento na compreensão do conceito de inteligência. Mencionam esses autores que crianças mais novas tendem a superestimar suas capacidades e não compreendem a ideia de que o esforço pode gerar mudanças na inteligência, enquanto crianças mais velhas são mais inclinadas a fazer autojulgamentos de capacidade mais realistas e a acreditar que o esforço interage com a inteligência.

Considerando a escassez de pesquisas nacionais acerca da motivação para aprender e a importância de conhecê-la melhor em nosso meio, o presente estudo teve como objetivo investigar: (a) as orientações motivacionais; (b) as crenças educacionais acerca dos conceitos de esforço, inteligência e sorte; (c) as crenças sobre as condições necessárias para a obtenção do sucesso na escola; e (d) as expectativas futuras dos alunos quanto à superação de um desempenho acadêmico ruim, de 120 alunos brasileiros do Ensino Fundamental. As relações dessas variáveis entre si e 
com o desempenho acadêmico, o gênero e a série escolar dos alunos também foram examinadas.

\section{MÉTODO}

\section{Participantes}

Participaram do presente estudo 120 alunos, sendo metade da amostra de terceiras séries e a outra metade, de quintas séries do Ensino Fundamental de escolas públicas estaduais do Interior de São Paulo/Brasil. Os estudantes eram de ambos os sexos, de idades entre oito e catorze anos. O desempenho acadêmico dos alunos foi avaliado com base no Teste de Desempenho Escolar/TDE (Stein, 1994) e nas informações sobre a ocorrência de repetências em sua trajetória escolar, obtidas por meio de uma entrevista estruturada com os alunos. A tabela 1 apresenta a distribuição dos alunos por série, gênero, repetência e resultados no TDE.

Tabela 1. Distribuição em Porcentagem dos Alunos por Série, Gênero, Repetência e Resultados no TDE

\begin{tabular}{cccccccc}
\hline \multirow{2}{*}{ Série } & $\begin{array}{c}N \text { total de } \\
\text { alunos }\end{array}$ & \multicolumn{2}{c}{ Gênero } & \multicolumn{2}{c}{ Repetência } & \multicolumn{2}{c}{ TDE } \\
\cline { 3 - 8 } & 60 & Fem. & Masc. & Não & Sim & Inferior & Médio \\
\hline $3^{3^{\mathbf{a}}}$ & 60 & 50,0 & 50,0 & 68,3 & 31,7 & 91,7 & 8,3 \\
$5^{-\mathbf{a}}$ & 56,7 & 43,3 & 80,0 & 20,0 & 83,3 & 16,7 \\
\hline
\end{tabular}

\section{Instrumentos}

Os dados foram obtidos por meio da Entrevista Estruturada de Crenças Educacionais e Orientações Motivacionais do Aluno - ECO-A, desenvolvida por Boruchovitch e Martini (2005). Esse instrumento é composto de itens para a obtenção dos dados demográficos, de vinte e três questões fechadas, relativas às crenças educacionais, e de onze questões fechadas, referentes às orientações motivacionais dos alunos. O trabalho de elaboração, revisão e refinamento do instrumento foi baseado na literatura da área (Piccinini, 1989; Ryan \& Deci, 2000; Weiner, 1985) e também nos resultados obtidos por meio de um estudo piloto. $\mathrm{O}$ instrumento completo encontra-se em Martini (1999).

Nas questões referentes às crenças educacionais, os alunos deveriam responder simplesmente sim ou não às questões ou escolher um item de uma lista de possíveis respostas. As questões de 1 a 18 avaliam a compreensão dos alunos acerca da importância, da localização (interna ou externa), da natureza (inata ou adquirida) e da estabilidade dos conceitos de esforço, inteligência e sorte. São exemplos dessas questões: (a) Você acha que o esforço é importante para alguém tirar boas notas na escola? (b) Você acha que uma pessoa esforçada é sempre esforçada ou depende da situação?

As questões 19 e 20 avaliam a interpretação dos alunos sobre a afetividade do professor em relação ao aluno e a influência dessa afetividade sobre o desempenho acadêmico desse aluno; por exemplo: (a) Você acha que seus professores gostam de você? e (b) Você acha que os professores gostarem ou não de você interfere nas suas notas? As questões de 21 a 23 verificam a interpretação dos alunos sobre o que é necessário para alcançar o sucesso na escola e suas expectativas quanto à superação de um desempenho ruim; por exemplo: (a) Você acha que pode recuperar uma nota ruim numa próxima avaliação?

Nas questões sobre as orientações motivacionais, os alunos também deveriam responder sim ou não às perguntas, ou escolher um item de uma lista de possíveis respostas. Essas questões versam sobre: a) aspectos que contribuem para a caracterização de um aluno como intrínseca ou extrinsecamente motivado, ou seja, a importância que ele atribui às expectativas dos outros em relação ao seu desempenho escolar; b) preferências dos alunos em relação às tarefas (simples ou mais complexas e desafiantes); (c) o esforço em tarefas com ou sem recompensa externa, entre outras. Um exemplo dessas questões é: $O$ que é mais importante para você: tirar boas notas em alguma matéria ou sentir que está entendendo a matéria quando estuda?

Para avaliação do desempenho escolar dos alunos utilizou-se o Teste de Desempenho Escolar de Stein (TDE). O TDE é um instrumento elaborado a partir da realidade escolar brasileira, composto por três subtestes sob a forma de escalas, para avaliação das capacidades de escrita, aritmética e leitura de escolares de primeira a sexta séries do Ensino Fundamental.

\section{Procedimentos de coleta de dados}

Os instrumentos foram aplicados individualmente em uma sala de aula cedida pela escola. Inicialmente, os objetivos da pesquisa foram apresentados pela pesquisadora aos alunos cujos pais haviam autorizado a participação, bem como lhes foi assegurado que a participação teria caráter confidencial e não interferiria 
em sua avaliação escolar. $\mathrm{O}$ anonimato de todos os participantes foi preservado.

\section{Tratamento dos dados}

Os dados do TDE foram analisados conforme as instruções existentes no manual de aplicação e avaliação do instrumento. As respostas obtidas por meio da ECO-A e do TDE foram objeto de análise estatística descritiva (frequência e porcentagem) e inferencial (Teste do Qui-Quadrado).
Identificou-se que a maioria dos alunos deste estudo foi avaliada como tendo uma orientação motivacional predominantemente intrínseca $(77,5 \%)$, enquanto somente $22,5 \%$ dos alunos foram avaliados como extrinsecamente motivados. Embora não se tenham constatado diferenças estatisticamente significativas, pode-se verificar uma ligeira predominância da orientação motivacional intrínseca em alunos da terceira série, do gênero feminino, repetentes e com desempenho médio no TDE. A motivação extrínseca prevaleceu entre os alunos da terceira série, do gênero masculino, não repetentes e com desempenho inferior no TDE (Tabela 2).

\section{RESULTADOS}

Tabela 2. Distribuição em Porcentagem das Orientações Motivacionais dos Alunos por Série, Gênero, Repetência e Desempenho no TDE

\begin{tabular}{cccccccccc}
\hline \multirow{2}{*}{$\begin{array}{c}\text { Orientações } \\
\text { Motivacionais }\end{array}$} & \multirow{2}{*}{ Total } & \multicolumn{2}{c}{ Série } & \multicolumn{2}{c}{ Gênero } & \multicolumn{2}{c}{ Repetência } & \multicolumn{2}{c}{ TDE } \\
\cline { 3 - 11 } & & $\mathbf{3}^{\mathbf{a}}$ & $\mathbf{5}^{\mathbf{a}}$ & Fem. & Masc. & Sim & Não & Inferior & Médio \\
\hline Intrínseca & 77,5 & 81,7 & 73,3 & 78,1 & 76,8 & 80,6 & 76,4 & 75,2 & 9,3 \\
Extrínseca & 22,5 & 28,3 & 26,7 & 21,9 & 23,2 & 19,4 & 23,6 & 24,8 & 6,7 \\
\hline
\end{tabular}

A análise da tabela 3 permite verificar que, para os alunos, o esforço e a inteligência são fatores altamente importantes para o sucesso escolar (100\%, respectivamente). Constata-se também que tanto o esforço quanto a inteligência foram considerados pelos alunos predominantemente como aspectos adquiridos ao longo da vida, internos e instáveis $(80,8 \%, 95,8 \%, 76,6 \%$ e 78,3\%, 90,8\%, 725\%, respectivamente). A sorte, por sua vez, foi considerada importante para o sucesso escolar por $81,7 \%$, uma variável adquirida $(73,3 \%)$, interna $(71,7 \%)$ e instável $(82,5 \%)$.

Tabela 3. Distribuição em Porcentagem da Avaliação dos Conceitos de Esforço (E), Inteligência (I) e Sorte (S) na Amostra Total e por Série, Gênero, Repetência, Desempenho no TDE e Orientações Motivacionais, Quanto à sua Importância para o Sucesso Escolar, a Natureza inata ou Adquirida, Localização e Estabilidade.

\begin{tabular}{|c|c|c|c|c|c|c|c|c|c|c|c|c|}
\hline & \multirow{2}{*}{ Avaliação } & \multirow{2}{*}{ Total } & \multicolumn{2}{|c|}{ Série } & \multicolumn{2}{|c|}{ Gênero } & \multicolumn{2}{|c|}{ Repetência } & \multicolumn{2}{|c|}{ TDE } & \multicolumn{2}{|c|}{ OM } \\
\hline & & & $3^{\mathrm{a}}$ & $5^{-a}$ & Fem. & Masc. & Não & Sim & I & M & I & $\mathbf{E}$ \\
\hline \multirow{7}{*}{$\mathrm{E}$} & Importante* & 100 & 100 & 100 & 100 & 100 & 100 & 100 & 100 & 100 & 100 & 100 \\
\hline & Inato & 19,2 & 13,3 & 25,0 & 15,6 & 23,2 & 20,2 & 16,1 & 19,0 & 20,0 & 20,4 & 14,8 \\
\hline & Adquirido & 80,8 & 86,7 & 75,0 & 84,4 & 76,8 & 79,8 & 83,9 & 81,0 & 80,0 & 79,6 & 85,2 \\
\hline & Interno & 95,8 & 95,0 & 96,7 & 95,3 & 96,4 & 93,6 & 93,5 & 95,2 & 100 & 94,6 & 100 \\
\hline & Externo & 4,2 & 5,0 & 3,3 & 4,7 & 3,6 & 3,4 & 6,5 & 4,8 & 0,0 & 5,4 & 0,0 \\
\hline & Estável & 23,3 & 23,3 & 23,3 & 18,8 & 28,6 & 19,1 & 35,5 & 24,8 & 13,3 & 22,6 & 25,9 \\
\hline & Instável & 76,7 & 76,7 & 76,7 & 81,3 & 71,4 & 80,9 & 64,5 & 75,2 & 86,7 & 77,4 & 74,1 \\
\hline \multirow{7}{*}{ I } & Importante* & 100 & 100 & 100 & 100 & 100 & 100 & 100 & 100 & 100 & 100 & 100 \\
\hline & Inata & 21,7 & 20,0 & 23,3 & 18,8 & 25,0 & 25,8 & 9,7 & 20,0 & 33,3 & 19,4 & 29,6 \\
\hline & Adquirida & 78,3 & 80,0 & 76,7 & 81,3 & 75,0 & 74,2 & 90,3 & 80,0 & 66,7 & 80,6 & 70,4 \\
\hline & Interna & 90,8 & 88,3 & 93,3 & 92,2 & 89,3 & $94,4 * *$ & 80,6 & 89,5 & 100 & 91,4 & 88,9 \\
\hline & Externa & 9,2 & 11,7 & 6,7 & 7,8 & 10,7 & 5,6 & $19,4 * *$ & 10,5 & 0 & 8,6 & 11,1 \\
\hline & Estável & 27,5 & 26,7 & 28,3 & 18,8 & $37,5 * *$ & 30,3 & 19,4 & 28,6 & 20,0 & 26,9 & 29,6 \\
\hline & Instável & 72,5 & 73,3 & 71,7 & $81,3 * *$ & 62,5 & 69,7 & 80,6 & 71,4 & 80,0 & 73,1 & 70,4 \\
\hline \multirow{8}{*}{ S } & Importante & 81,7 & $95,0 * * *$ & 68,3 & 87,5 & 75,0 & 82,0 & 80,6 & 82,9 & 73,3 & 79,6 & 88,9 \\
\hline & NãoImport, & 18,3 & 5,0 & 31,7 & 12,5 & 25,0 & 18,0 & 19,4 & 17,1 & 26,7 & 20,4 & 11,1 \\
\hline & Inata & 26,7 & 30,0 & 23,3 & 28,1 & 25,0 & 29,2 & 19,4 & 27,6 & 20,0 & 30,1 & 14,8 \\
\hline & Adquirida & 73,3 & 70,0 & 76,7 & 71,9 & 75,0 & 70,8 & 80,6 & 72,4 & 80,0 & 69,9 & 85,2 \\
\hline & Interna & 71,7 & 76,7 & 66,7 & 76,6 & 66,1 & 74,2 & 64,5 & 73,3 & 60,0 & 74,2 & 63,0 \\
\hline & Externa & 28,3 & 23,3 & 33,3 & 23,4 & 33,9 & 25,8 & 35,5 & 26,7 & 40,0 & 25,8 & 37,0 \\
\hline & Estável & 17,5 & 15,0 & 20,0 & 18,8 & 16,1 & 15,7 & 22,6 & 19,0 & 6,7 & 15,1 & 25,9 \\
\hline & Instável & 82,5 & 85,0 & 80,0 & 81,3 & 83,9 & 84,3 & 77,4 & 81,0 & 93,3 & 84,9 & 74,1 \\
\hline
\end{tabular}

Nota: $\quad *$ Não foram realizadas provas de Qui-quadrado com estas respostas, tendo em vista que o Esforço e a Inteligência foram considerados pela totalidade da amostra como uma atribuição importante, caracterizando-se assim como variáveis constantes. **p $\leq 0.01 ; * * * \mathrm{p} \leq 0.001$. 
Algumas diferenças significativas foram encontradas na avaliação dos conceitos de esforço, inteligência e sorte pelos alunos em relação à série, ao gênero e à repetência. Os alunos de terceira série concebem a sorte como um fator muito mais importante para o sucesso escolar, se comparados aos alunos de quinta série $\left(\mathrm{X}^{2}(1)=14.249 ; \mathrm{p} \leq 0.001\right)$. As meninas percebem a inteligência como um atributo instável, enquanto meninos a avaliaram como estável $\left(\mathrm{X}^{2}(1)=5.266 ; \mathrm{p} \leq 0.01\right)$. Alunos não repetentes veem a inteligência como um construto interno, enquanto alunos repetentes a imaginam como um fator externo ao indivíduo $\left(\mathrm{X}^{2}(1)=5.211 ; \mathrm{p} \leq 0.01\right)$.

$\mathrm{Na}$ tabela 4 pode-se verificar a distribuição das respostas dos alunos sobre as demais questões relativas às crenças educacionais. Pode-se constatar que, quando indagados se o professor gosta deles, $76,7 \%$ responderam que sim, mas afirmaram também que o fato de os professores gostarem ou não gostarem deles não interfere em suas notas na escola (70,0\%). Para a maioria dos alunos, é necessário ter a ajuda de alguém para se obter sucesso na escola $(65,0 \%)$ e é possível melhorar um desempenho acadêmico ruim $(91,7 \%)$. A maioria acredita, ainda, que o esforço é o suficiente para se ir bem na escola $(59,2 \%)$, muito embora não pense que seja possível aprender rápido sem esforço $(58,3 \%)$ e que a inteligência é o suficiente para um aluno conseguir aprender $(69,2 \%)$.

Tabela 4. Distribuição em Porcentagem das Respostas dos Alunos Sobre as Questões Referentes às Crenças Gerais dos Alunos

\begin{tabular}{lcc}
\hline Crenças educacionais & Sim & Não \\
\hline Professores gostam de você & 76,7 & 23,3 \\
Professores gostarem de você interfere nas notas & 30,0 & 70,0 \\
Necessário a ajuda de alguém para ir bem na escola & 65,0 & 35,0 \\
Acredita que pode melhorar uma nota ruim & 91,7 & 8,3 \\
Esforço é suficiente para se ir bem na escola & 59,2 & 40,8 \\
Acredita que aprende rápido sem esforço & 41,7 & 58,3 \\
Inteligência é o suficiente para um aluno aprender & 30,8 & 69,2 \\
\hline
\end{tabular}

Pôde-se verificar, também, que, quando questionados se os professores gostam deles, meninas e alunos não repetentes respondem significativamente mais que sim, se comparados aos meninos e alunos repetentes $\left(\mathrm{X}^{2}(1)=6.589 ; \mathrm{p} \leq 0.01\right.$ e $\mathrm{X}^{2}(1)=5.524$; $\mathrm{p} \leq 0.01$, respectivamente). Alunos repetentes $\mathrm{e}$ extrinsecamente motivados concordaram significativamente mais com a afirmação de que os professores gostarem ou não deles interfere nas notas obtidas na escola $\left(\mathrm{X}^{2}(1)=6.729 ; \mathrm{p} \leq 0.001\right.$ e $\mathrm{X}^{2}(1)=$ 7.922; $\mathrm{p} \leq 0.001)$.

Tabela 5. Porcentagem de Concordância ou Respostas "Sim" dos Alunos em Relação às Crenças Educacionais por Gênero, Série, Repetência, Resultados do TDE e Orientações Motivacionais

\begin{tabular}{ccccccccccc}
\hline \multirow{2}{*}{ Crenças } & \multicolumn{2}{c}{ Gênero } & \multicolumn{2}{c}{ Série } & \multicolumn{2}{c}{ Repetência } & \multicolumn{2}{c}{ TDE } & \multicolumn{1}{c}{ OM } \\
\cline { 2 - 11 } & Fem, & Masc, & $\mathbf{3}^{\mathbf{a}}$ & $\mathbf{5}^{\mathbf{a}}$ & Não & Sim & I & M & I & E \\
\hline PG & $85,9 * *$ & 66,1 & 71,7 & 81,7 & $82,0^{* *}$ & 61,3 & 76,2 & 80,0 & 78,5 & 70,4 \\
PGIN & 23,4 & 37,5 & 30,0 & 30,0 & 23,6 & $48,4 * * *$ & 29,5 & 33,3 & 23,7 & $51,9 * * *$ \\
NAN & 67,2 & 62,5 & 71,7 & 58,3 & 61,8 & 74,2 & 63,8 & 73,3 & 67,7 & 55,6 \\
PMD & 92,2 & 91,1 & 86,7 & $96,7 *$ & $96,6 * *$ & 77,4 & 90,5 & 100,0 & 90,3 & 96,3 \\
ESE & 51,6 & 67,9 & 60,0 & 58,3 & 57,3 & 64,5 & 59,0 & 60,0 & 55,9 & 70,4 \\
ASE & 40,6 & 42,9 & $51,7 * *$ & 31,7 & 41,6 & 41,9 & 41,0 & 46,7 & 44,1 & 33,3 \\
ISA & 23,4 & 39,3 & $45,0^{* * *}$ & 16,7 & 27,0 & 41,9 & 31,4 & 26,7 & 25,8 & $48,1 * *$ \\
\hline
\end{tabular}

Nota: $\quad$ PG = Professores gostam de você; PGIN = Professores gostarem de você interfere nas notas; NAN = Necessário ajuda de alguém para ir bem na escola; PMD = Pode melhorar o desempenho; ESSE = Esforço é suficiente para ir bem na escola; ASE = Aprende rápido sem esforço; ISA = Inteligência é suficiente para aprender; *p.0.05;**p $\leq 0.01 ; * * * \mathrm{p} \leq 0.001$

Quando comparados aos alunos com história de repetência, os alunos não repetentes acreditam significativamente mais que podem melhorar o desempenho acadêmico ruim $\left(\mathrm{X}^{2}(1)=11.107\right.$; $\mathrm{p} \leq 0.001$ ), do mesmo modo que os alunos de quinta série, quando comparados aos de terceira $\left(\mathrm{X}^{2}(1)=\right.$ 3.927; $\mathrm{p} \leq 0.05$ ). Os alunos de terceira série, por sua vez, demonstraram acreditar que é possível aprender rápido sem esforço $\left(\mathrm{X}^{2}(1)=4.937 ; \mathrm{p} \leq 0.01\right)$ e que a inteligência é o suficiente para se aprender na escola $\left(\mathrm{X}^{2}(1)=11.293 ; \mathrm{p} \leq 0.001\right)$. Os alunos extrinsecamente motivados também consideraram a inteligência um fator suficiente para que a aprendizagem seja bem-sucedida $\left(\mathrm{X}^{2}(1)=4.898\right.$; $\mathrm{p} \leq 0.01)$.

\section{DISCUSSÃO}

Os resultados do presente estudo demonstram o predomínio da orientação motivacional intrínseca nos alunos. É inegável que o padrão motivacional intrínseco seja altamente apropriado para a aprendizagem dos estudantes (Gottfried, Marcoulides, 
Gottfried, Oliver \& Guerin, 2007), resultado que também foi confirmado no presente estudo, pela prevalência dessa orientação motivacional entre os alunos de melhor desempenho escolar no TDE.

Se, por um lado, o predomínio da motivação intrínseca entre os participantes da presente investigação parece um dado surpreendente e possibilita questionamentos como: ter-se-iam encontrado alunos excepcionalmente motivados nessa pesquisa? Será que o dado encontrado foi decorrente de um possível erro de medida, já que o instrumento utilizado pode ter sido simplista? Será que os participantes deram respostas socialmente desejáveis ao entrevistador? Por outro lado, os resultados encontrados no presente estudo parecem dar plausibilidade aos achados de outras pesquisas brasileiras, não só com crianças do ensino fundamental, empregando instrumentos diversos (Arcas, 2003; Boruchovitch, 2006; Boruchovitch, 2008; Manzini, 2006 Neves \& Boruchovitch, 2004), mas também com estudantes universitários (Zenorine \& Santos, 2003).

Uma possível explicação para esse achado pode estar relacionada com o fato de que, embora a Teoria da Integração Organísmica tenha revelado as nuanças da motivação para aprender, na literatura, lamentavelmente, ainda predominam estudos mais voltados para a compreensão da motivação dos estudantes nas formas mais extremas do continuum (motivação intrínseca e extrínseca), quer em nível internacional (Lepper, Corpus \& Iyengar, 2005; Gottfried, 1990; Gottfried, 1985; 2001; 2004; Gottfried, Gottfried, Cook \& Morris, 2005; Gottfried, Marcoulides, Gottfried, Oliver \& Guerin, 2007), quer em nível nacional (Boruchovitch, 2006; Boruchovitch, 2008; Manzini, 2006; Neves \& Boruchovitch; 2004; Neves \& Boruchovitch, 2007).

A escassez desses estudos pode ser decorrente de vários fatores, entre eles as expectativas de que o continuum seja difícil de ser examinado em crianças e/ou a ausência de instrumentos capazes de mensurar e diferenciar todas as nuanças do continuum motivacional. Acrescenta-se a essas ponderações, como descrito em Neves e Boruchovitch (2007), o fato de que, ao que tudo indica, os instrumentos nacionais têm conseguido medir apenas uma orientação geral do aluno para a aprendizagem, e não sua motivação para aprender, específica e contextualizada.

Além disso, em revisão da literatura da área, Neves e Boruchovitch (2004) identificaram resultados de pesquisa que retratam a opinião dos professores sobre o impacto negativo que o regime de progressão continuada exerce sobre a motivação do aluno. É possível, nesse caso, aventar ainda a hipótese de que os professores considerem seus alunos muito mais desmotivados do que realmente o são, já que dados provenientes da pesquisa de Neves e Boruchovitch (2004) revelaram que os estudantes reconheciam a importância e o valor do estudo para suas vidas, muito embora não conhecessem nem compreendessem bem o regime da progressão continuada.

Essas considerações podem esclarecer as razões pelas quais a quase totalidade dos alunos repetentes desta pesquisa demonstrou ser intrinsecamente motivada, contrariando as hipóteses anteriores referentes à relação entre a repetência (como um fator do desempenho acadêmico) e a orientação motivacional dos alunos. Uma possível incompatibilidade entre a orientação motivacional desses alunos (intrínseca), somada à experiência de fracasso e à estrutura motivacional de sala de aula (tradicionalmente extrínseca), pode ter comprometido o envolvimento, o esforço e a persistência nas tarefas acadêmicas dos alunos repetentes deste estudo, resultando em um desempenho escolar abaixo do esperado (Guimarães, 2004). Precisar o tipo de motivação do estudante brasileiro tem importantíssimas implicações educacionais e, sendo assim, recomenda-se que esse constructo seja objeto de um maior aprofundamento teórico e metodológico, em futuras investigações.

Uma questão também importante, porém controvertida na literatura, é se a orientação motivacional intrínseca tende a aumentar, permanecer constante ou declinar com o avançar da série escolar e da idade. Neste sentido, o presente estudo corrobora resultados de pesquisas internacionais (Lepper, Corpus \& Iyengar, 2005) e da maioria das investigações nacionais (Arcas, 2003; Boruchovitch, 2006; Martini \& Boruchovitch, 2004), ao constatar um ligeiro declínio da orientação motivacional intrínseca ao longo dos anos. Dadas as implicações educacionais também desse resultado, entende-se que é fundamental que pesquisadores dessa área focalizem esse aspecto em seus estudos.

A compreensão da inteligência, do esforço e da sorte como fatores importantes para o sucesso escolar, internos, adquiridos ao longo da vida e instáveis, revela que os alunos deste estudo apresentam, em linhas gerais, crenças positivas para a aprendizagem. A internalidade e a instabilidade das causas favorecem a percepção de que é possível aprimorar essas características e superar desempenhos malsucedidos, contribuindo para uma aprendizagem de qualidade autorregulada, na qual o indivíduo pode perceber-se capaz de direcionar suas cognições e comportamentos 
em direção à realização de seus objetivos (Cleary \& Zimmerman, 2004).

Resultados semelhantes a esses foram encontrados na literatura nacional por Boruchovitch (2001) com os alunos de mesma faixa etária e nível socioeconômico, bem como estão em consonância com as evidências de que crianças a partir de oito anos de idade passam a compreender que o esforço e a inteligência não são conceitos unificados e, ainda, que o esforço pode gerar mudanças na inteligência, demonstrando autojulgamentos de capacidade mais realistas e crenças mais complexas de que o esforço pode e deve interagir com a inteligência (Blumenfeld, Pintrich \& Hamilton,1986).

Não obstante, os meninos deste estudo consideraram a inteligência um fator estável e os alunos repetentes a consideraram externa, revelando uma compreensão da inteligência como um traço imutável, fixo e difícil de ser alterado. Essa interpretação pode influenciar negativamente o desempenho escolar desses alunos, pois atribuir ao fracasso causas estáveis e externas ao indivíduo pode levar a uma diminuição da motivação, da expectativa de sucesso futuro, da autoestima e do autoconceito, bem como prejudicar o desenvolvimento da autorregulação da aprendizagem (Blackwell, Trzesniewski \& Dweck, 2007; Cleary \& Zimmerman, 2004; Martini \& Boruchovitch, 2004).

Em relação às demais crenças educacionais investigadas, verificou-se que a maioria dos alunos deste estudo demonstrou crenças altamente positivas para a aprendizagem escolar. Acreditam que seus professores gostam deles, mas que esse fator não interfere nas notas obtidas na escola, bem como creem que é necessária a ajuda de alguém para se obter o sucesso escolar, reconhecendo o caráter social e afetivo do processo de ensino-aprendizagem (Coll \& Martí, 2004). A maior parte dos alunos também demonstra boas expectativas quanto à superação de um fracasso escolar, concebendo o esforço, e não somente a inteligência, como fatores importantes para a aprendizagem bem-sucedida.

Por outro lado, novamente os meninos, os alunos repetentes e extrinsecamente motivados apresentaram um conjunto de crenças menos apropriado à motivação para a aprendizagem e ao sucesso escolar. Esses alunos acreditam que seus professores não gostam deles e concordaram significativamente mais com o fato de que os professores gostarem ou não deles interfere nas notas obtidas na escola. Por outro lado, os alunos não repetentes e de quinta série acreditam significativamente mais que podem melhorar um desempenho acadêmico ruim, revelando expectativas mais positivas de sucesso futuro, quando comparados aos demais alunos.

Diferenças no padrão de crenças educacionais relacionadas ao gênero e ao desempenho acadêmico também foram encontradas em outras pesquisas brasileiras com crianças da mesma faixa etária e nível socioeconômico, nas quais meninas e alunos de alto desempenho escolar apresentaram um padrão atribucional mais apropriado à motivação e ao sucesso escolar, enquanto os meninos demonstraram crenças frequentemente relacionadas ao baixo autoconceito, à baixa autoestima e ao desamparo adquirido (Boruchovitch, 2001; Martini \& Boruchovitch, 2004). Esforços futuros devem também ser direcionados para uma melhor compreensão do impacto que variáveis como gênero e repetência possam ter nas orientações motivacionais e nas crenças educacionais de nossos estudantes.

Em relação às diferenças nas crenças dos alunos com o avanço da escolaridade, constatou-se que as crianças mais novas, da terceira série, acreditam significativamente mais que a inteligência é o suficiente para se aprender na escola e, consequentemente, que podem aprender rápido, sem esforço. Esses resultados confirmam os dados da literatura, ao demonstrarem que crianças mais novas superestimam suas capacidades e não compreendem a ideia de que o esforço pode gerar mudanças na inteligência. Crianças mais velhas, por sua vez, são mais inclinadas a fazer autojulgamentos de capacidade mais realistas e a acreditar que o esforço interage com a inteligência para a realização bem-sucedida de uma tarefa acadêmica (Blumenfield, Pintrich \& Hamilton, 1986).

Em síntese, os resultados deste estudo demonstram o predomínio da orientação motivacional intrínseca nos alunos e de um padrão de crenças altamente apropriado à motivação e ao desempenho escolar. Alguns resultados reforçam a necessidade de se compreenderem mais profundamente a motivação e as variáveis demográficas e motivacionais dos alunos, com base em avanços metodológicos, dada a relevância das implicações educacionais envolvidas, bem como apontam a necessidade de planejamento de intervenções mais focalizadas. As crenças de meninos, alunos repetentes e extrinsecamente motivados, que vêm sendo confirmadas na literatura como menos apropriadas ao desenvolvimento socioemocional, cognitivo, afetivo e motivacional desses alunos merecem ser alvo de atenção especial.

\section{CONSIDERAÇÕES FINAIS}

Pesquisas têm demonstrado que a motivação dos alunos constitui uma das maiores preocupações dos 
professores (Bzuneck, 2004); no entanto, apesar dos avanços obtidos nos últimos anos, a pesquisa motivacional no Brasil ainda traz resultados fragmentados e difusos, o que tem justificado o constante investimento de esforços nessa área (Martini \& Boruchovitch, 2004). Neste estudo, essa preocupação foi encaminhada por meio da análise das orientações motivacionais e de um conjunto de crenças educacionais de alunos brasileiros do Ensino Fundamental.

Não resta dúvida de que a compreensão da motivação como um processo mediado pelas variáveis cognitivas e afetivas - e não mais como um traço estável de personalidade - constitui um avanço importante no campo educacional. As crenças pessoais são passíveis de mudança e a realização de intervenções com foco na alteração de crenças inadequadas pode gerar mudanças positivas na motivação e na aprendizagem dos alunos (Martini \& Boruchovitch, 2004). Não obstante, como ressalta Pajares (1992), quanto mais cedo uma crença é aprendida, maior será a dificuldade de alterá-la, o que justifica o investimento em conhecer e trabalhar as crenças dos alunos desde o início da escolarização.

Uma questão também fundamental é a coexistência das orientações motivacionais intrínseca e extrínseca. Muito embora a motivação intrínseca seja uma meta educacional altamente desejável, em sua maioria, as atividades escolares não são intrinsecamente orientadas, sendo igualmente importante a compreensão da orientação motivacional extrínseca, que pode variar fortemente quanto ao grau de autonomia do indivíduo no processo motivacional.

De acordo com Ryan e Deci (2000), os alunos podem apresentar um desempenho extrinsecamente motivado, que seja marcado por uma atitude positiva, pela vontade e pela internalização do valor de uma tarefa. Essa forma qualitativamente diferente de motivação extrínseca pode gerar desempenhos diferentes e um melhor engajamento na escola, o que faz dessa forma de motivação extrínseca uma meta educacional também desejável.

Conclui-se que existem múltiplas formas e caminhos de se promover uma motivação positiva nos alunos em contexto de aprendizagem. É fundamental que esse conhecimento seja traduzido em práticas pedagógicas que propiciem a motivação do aluno. É importante reconhecer, por outro lado, que nem sempre os professores estão preparados para isso e esse conhecimento pode e deve ser utilizado por psicólogos e educadores na capacitação desses professores, em busca de um contexto pedagógico mais favorável ao sucesso escolar.
Cabe, finalmente, ressaltar que um importante desdobramento da presente pesquisa foi a oportunidade de ter apresentado os resultados aqui encontrados ao diretor, aos professores e ao coordenador da escola participante, o que lhes proporcionou a possibilidade não só de compreender mais profundamente a motivação de seus estudantes, mas também de discutir os impactos desse conhecimento na prática docente, diminuindo, assim, o hiato existente entre a produção do conhecimento científico e a prática pedagógica.

\section{REFERÊNCIAS}

Arcas, P. H. (2003). Avaliação da aprendizagem no regime de progressão continuad, o que dizem os alunos. Dissertação de Mestrado, Faculdade de Educação, Universidade de São Paulo, São Paulo.

Bandura, A. (1982). Self-efficacy mechanism in human agency. American Psychologist, 37, 122-147.

Blackwell, L., Trzesniewski, K. \& Dweck, C. S. (2007). Implicit theories of intelligence predict achievement across an adolescent transition, A longitudinal study and an intervention. Child Development, 78, 246-263.

Blumenfield, P. C., Pintrich, P. R. \& Hamilton, V. L. (1986). Children's concepts of ability, effort and conduct. American Educational Research, 28, 95-104.

Boruchovitch, E. (2001). Conhecendo as Crenças sobre Inteligência, Esforço e Sorte de Alunos Brasileiros em Tarefas Escolares. Psicologia. Reflexão e Crítica, 14(3), 461-467.

Boruchovitch, E. (2006). Estratégias de aprendizagem, uma análise à luz das variáveis demográficas e motivacionais. Tese (LivreDocência), Faculdade de Educação, Universidade Estadual de Campinas, Campinas.

Boruchovitch, E. (2008). Motivação para aprender de estudantes em curso de formação de professores. Revista Educação-PUC-RS, 31(1), 30-38.

Boruchovitch, E., \& Martini, M. L. (2005). A construção de um instrumento para avaliação das atribuições de causalidade em contexto escolar. Anais do II Congresso Brasileiro de Avaliação Psicológica (p. 18). Instituto Brasileiro de Avaliação Psicológica, Gramado, Brasil.

Bzuneck, J. A. (2004). A motivação do aluno, Aspectos introdutórios. Em E. Boruchovitch \& J. A. Bzuneck (Eds.), Motivação do aluno, Contribuições da psicologia contemporânea ( $3^{\mathrm{a}}$ ed., pp. 09-36). Petrópolis, RJ, Vozes.

Cleary, T. J. \& Zimmerman, B. J. (2004). Self-regulation empowerment program: A school-based program to enhance selfregulated and self-motivated cycles of student learning. Psychology in the Schools, 41, 537-550.

Coll, C. \& Martí, E. (2004). Aprendizagem e desenvolvimento, a concepção genético-cognitiva da aprendizagem. Em C. Coll, J. Palácios \& A. Marchesi (Eds.). Desenvolvimento Psicológico e Educação. Psicologia da Educação (pp. 45-59). Porto Alegre, Artes Médicas. 
Dweck, C. \& Bempechat, J. (1983). Children's theory of intelligence, Consequences of learning. In Paris, S., Olson, G. \& Stevenson, H. (Eds). Learning and motivation in the classroom. Hillsdale, NJ, Erlbaum, p.239-255.

Gottfried, A. E. \& Gottfried, A. W. (2004). Toward the development of a conceptualization of gifted motivation. Gifted Child Quarterly, 48, 121-132.

Gottfried, A. E. (1985). Academic intrinsic motivation in elementary and junior high school students. Journal of Educational Psychology, 77(6), 631-645.

Gottfried, A. E. (1990). Academic intrinsic motivation in young elementary school children. Journal of Educational Psychology, 82(3), 525-538.

Gottfried, A. E., Fleming, J. S. \& Gottfried, A.W. (2001). Continuity of academic intrinsic motivation from childhood through late adolescence, A longitudinal study. Journal of Educational Psychology, 93(1), 3-13.

Gottfried, A. E., Marcoulides, G. A., Gottfried, A. W., Oliver, P. H. \& Guerin, D. W. (2007). Multivariate latent change modeling of developmental decline in academic intrinsic math motivation and achievement, Childhood through adolescence. International Journal of Behavioral Development, 31(4), 317 - 327.

Gottfried, A. W., Gottfried, A.E., Cook, C. \& Morris, P. (2005). Educational characteristics of adolescents with gifted academic intrinsic motivation, A longitudinal study from school entry through early adulthood. Gifted Child Quarterly, 49, 172-186.

Guimarães, S. E. R. (2004). Motivação intrínseca, extrínseca e o uso de recompensas em sala de aula. Em E. Boruchovitch \& J. A. Bzuneck (Eds.), Motivação do aluno: Contribuições da psicologia contemporânea (3a ed., pp. 37-57). Petrópolis, RJ, Vozes.

Lepper, M. R., Corpus, J. H. \& Iyengar, S. (2005). Intrinsic and extrinsic motivation in the classroom, Age differences and academic correlates. Journal of Educational Psychology, 97(2), 184-196.

Manzini, C. H. G. (2006). Motivação no contexto escolar e desempenho acadêmico. Dissertação de mestrado. Faculdade de Educação, Unicamp, Campinas.

Martini, M. L. \& Boruchovitch, E. (2004). A teoria da atribuição de causalidade, Contribuições para a formação e atuação de educadores. Campinas, Alínea.
Martini, M. L. (1999). Atribuições de causalidade, crenças gerais e orientações motivacionais de crianças brasileiras. Dissertação de Mestrado não publicada. Faculdade de Educação. Universidade Estadual de Campinas, Campinas. São Paulo, Brasil.

Neves, E. R. C. \& Boruchovitch, E. (2004). A motivação de alunos no contexto da progressão continuada. Psicologia, Teoria $e$ Pesquisa, 20, 01, 77-85.

Neves, E. R. C. \& Boruchovitch, E. (2007). Escala de avaliação da motivação para aprender de alunos do ensino fundamental (EMA). Revista Psicologia Reflexão e Crítica, 20(3), 406-413.

Pajares, M. F (1992). Teacher's belief and educational research, Cleaning up a messy construct. Review of Educational Research, 62(3), 307-332.

Piccinini, C. A. (1989). Atribuições de causalidade em crianças, A alguns aspectos críticos. Psicologia: Teoria e Pesquisa, 5, 57-69.

Polydoro. Estudante universitário, características e experiências de formação. (pp. 67-86). Taubaté, Cabral Editora.

Reeve, J., Deci, E.L. \& Ryan, R.M. (2004). Self-determination theory: a dialetical framework for understanding sociocultural influences on student motivation. In Mcinerney, D. M. \& Van Etten, S. (Orgs.). Big theories revisited (pp 31-60). Greenwich, EUA: Information Age Publishing.

Ryan, R. M. \& Deci, E. L. (2000). Intrinsic and extrinsic motivations: Classic definitions and new directions. Contemporary Educational Psychology, 25, 54-67.

Stein, L. M. (1994). Teste de desempenho escolar. São Paulo, Casa do Psicólogo.

Weiner, B. (1985). An attributional theory of achievement motivation and emotion. Psychological Review, 92, 548-573.

Zenorini, R. P. C. \& Santos, A. A. A. (2003). A motivação e a utilização de estratégias de aprendizagem em universitários. Em E. Mercuri \& S. A. J.
Endereço para correspondência:
Mirella Paiva. Av. Epitácio Pessoa, 5076, Lagoa, CEP: 22471-001, Rio de Janeiro-RJ, Brasil. E-mail: evely@unicamp.br.
Recebido em 24/04/2008 Aceito em 16/10/2008 